\title{
Websurveys nos tempos de COVID-19
}

\author{
Web surveys in the time of COVID-19
}

Websurveys en tiempos de la COVID-19

Raquel Brandini De Boni 1

doi: 10.1590/0102-311X00155820

Viver e pesquisar nos tempos de COVID-19 pode ser semelhante à fase inicial do relacionamento amoroso de Florentino Ariza e Fermina Daza, no clássico O Amor nos Tempos do Cólera 1: estamos mais do que nunca utilizando a correspondência. Por correspondência entenda-se especificamente o uso do e-mail e das websurveys. Entre os diversos tipos de estudos que podem ser realizados utilizando a Internet - o livro Internet Research Methods 2 apresenta uma revisão didática para os que estão iniciando e vários exemplos interessantes para os mais experientes - aqui se discutem apenas as websurveys, também comumente chamadas de web-based surveys, e-surveys e on-line surveys. São brevemente apresentadas algumas das vantagens que podem ter influenciado sua popularização neste período (além da possibilidade de coletar dados a distância, uma vantagem clara quando existe a necessidade de distanciamento social), assim como os desdobramentos e questões éticas que precisam ser considerados durante seu planejamento e interpretação dos resultados.

Websurveys são estratégias usadas para a obtenção de dados primários, e vêm sendo realizadas desde os anos 1990. Diferentes áreas do conhecimento, incluindo as Ciências Sociais, Políticas, do Comportamento e da Comunicação, as têm utilizado e discutido desde então as possíveis limitações e necessidades de avanços metodológicos 3,4,5. De modo geral, websurveys podem ser realizadas utilizando-se cadastros de endereços de e-mail (membros de uma categoria profissional ou estudantes de uma universidade, por exemplo 6,7), painéis de usuários da Internet 8,9 ou a divulgação do link da pesquisa em websites e redes sociais 10,11. Considerando que cada uma dessas apresenta suas características, o foco será dado a websurveys que incluem voluntários recrutados em websites e redes sociais.

Uma rápida busca no PubMed/MEDLINE utilizando os termos "('websurvey' OR 'web-based survey' OR online survey) AND COVID-19", aproximadamente seis meses após o diagnóstico do primeiro caso de COVID-19 na China, encontra 146 artigos publicados. Nem de longe esse número representa o número total de websurveys que estão sendo conduzidas globalmente, mas deixa clara a primeira grande vantagem do método: a rapidez entre o planejamento e a publicação de resultados. Note-se que não estão sendo considerados resultados de relatórios, pesquisas de opinião ou quaisquer outros. Apenas artigos publicados em revistas científicas, que os selecionam por meio da avaliação de pares, na área da saúde e em uma única base bibliográfica. A velocidade na obtenção e divulgação de informações é particularmente importante em situações de crise. Um entendimento mínimo sobre percepção, crenças, conhecimento, comportamentos e problemas de saúde (física e mental) pode balizar novos estudos e intervenções quando não existem outros dados disponíveis. Somada à velocidade,
1 Instituto de Comunicação e Informação Científica e Tecnológica em Saúde, Fundação Oswaldo Cruz, Rio de Janeiro, Brasil.

\section{Correspondência} R. B. De Boni Instituto de Comunicação e Informação Científica e Tecnológica em Saúde, Fundação Oswaldo Cruz. Av. Brasil 4365, Rio de Janeiro, RJ 21040-360, Brasil.

raqueldeboni@gmail.com 
são notáveis os números de indivíduos incluídos (milhares ou dezenas de milhares de voluntários podem ser entrevistados em períodos tão curtos quanto 15 dias), bem como a abrangência geográfica e a possibilidade de atravessar fronteiras de forma praticamente imediata. Websurveys de abrangência nacional e mesmo as que incluem vários países podem gerar resultados em poucas semanas, especialmente quando o recrutamento é realizado por meio de redes sociais (como Facebook, Instagram, Twitter e WhatsApp, por exemplo).

Velocidade, grandes números e larga abrangência, porém, não garantem a validade externa (representatividade ou generalização dos resultados), que somente é atingida com a utilização de métodos probabilísticos de amostragem ou censos. Essa limitação é descrita desde as primeiras websurveys e diferentes grupos/instituições buscam desenvolver métodos de recrutamento, amostragem e análise para superá-la. Alguns pontos que tornam a tarefa mais árdua são: (1) a cobertura de Internet (por exemplo, a TIC Domicílios 201912 aponta que aproximadamente 74\% dos brasileiros utilizam a Internet, entretanto, a proporção é de $57 \%$ quando se consideram as classes socioeconômicas D e E; (2) ausência de um cadastro único de usuários da Internet; (3) a quantificação da não-resposta; (4) o viés de seleção; e (5) a possibilidade de um único usuário da Internet responder múltiplas vezes o questionário da pesquisa.

Para tornar uma longa estória curta: quando colocamos em um website/impulsionamos um banner com o link de pesquisa em uma rede social, não temos como assegurar o número exato de usuários que foram alcançados, tampouco suas características. Mesmo utilizando medidas de tráfego de web pages ou o alcance estimado pelas redes sociais, temos tão somente aproximações que podem não refletir a realidade. Por exemplo, quando um determinado IP (Internet Protocol) acessa uma página várias vezes, é possível que um mesmo usuário esteja retornando ou que o acesso tenha sido realizado por outra pessoa que utiliza o mesmo computador. Tanto o número quanto as características desses usuários definiriam a população da qual obtivemos uma amostra e em relação a qual desejaríamos fazer inferências. Além disso, essas são variáveis fundamentais para o cálculo das probabilidades de inclusão e da proporção e avaliação de não-resposta (ou seja, as informações necessárias para responder se os usuários que não participaram são semelhantes aos que participaram). Em seguida, temos a questão da autosseleção, ou seja, as características dos voluntários que participam do estudo podem estar relacionadas aos desfechos (superestimando ou subestimando prevalências e medidas de associação). Quando conhecemos previamente as associações, conseguimos minimamente discutir em que direção estaríamos errando. Por exemplo, em uma amostra composta principalmente por mulheres, esperamos que a prevalência de depressão seja superior à da população geral (pois a depressão é mais frequente entre mulheres). Porém, é preciso lembrar que existem variáveis que não conseguimos mensurar e que podem alterar os resultados de forma que não conseguimos identificar. Finalmente, temos a possibilidade de um participante responder mais de uma vez ao questionário. Em pesquisas anônimas (quando nenhuma forma de identificação do participante é coletada) é praticamente impossível garantir que um participante respondeu uma única vez. Na tentativa de aumentar a transparência, a possibilidade de interpretação dos resultados e reprodutibilidade de websurveys, em 2004 foi publicado um guideline, o The Checklist for Reporting Results of Internet E-Surveys (CHERRIES) 13. Embora não seja amplamente usado e a sugestão para evitar duplicidade (bloqueio do IP) não seja aceita universalmente 2 , todas essas considerações estão bem descritas e podem ajudar a comunidade científica a aumentar a qualidade e interpretação dos estudos.

Duas outras vantagens das websurveys também podem ter sido responsáveis por sua popularidade durante a pandemia: custo e facilidade de implantação. Sobre o custo, a título de exemplo, dois economistas australianos compararam o custo de uma websurvey e um inquérito postal (uma estratégia com inúmeras limitações, mas de baixo custo) entre indivíduos que visitaram a maior ilha de areia do mundo, a Ilha Fraser (Queensland, Austrália). Considerando que os autores compraram um software para a programação do questionário on-line, e excluindo o custo de seu próprio tempo de trabalho, cada questionário válido da websurvey custou USD 1,94 e os questionários válidos da pesquisa postal custaram cada um USD 8,13 14. Em relação à implantação, com o grande número, variedade e flexibilidade de softwares e servidores disponíveis para a programação e o armazenamento dos questionários on-line, as dificuldades iniciais de implantação foram superadas. Mesmo alguém sem nenhum conhecimento de linguagem de programação consegue colocar no ar um questionário simples. Essa 
última vantagem, além de tudo, deixa para trás a necessidade de planejar uma estratégia adicional para a entrada de dados (uma decisão central no planejamento de estudos off-line).

Entretanto, o melhor e mais bem programado software e o servidor mais seguro não garantem questionários consistentes e medidas acuradas. A construção e validação de questionários, especialmente aqueles que avaliam aspectos subjetivos e são autopreenchidos, são processos complexos que costumam demandar um estudo (ou vários) em si mesmo (estes estudos também podem ser realizados on-line, a propósito 15). Nesse sentido, a utilização de questionários validados previamente, quando disponíveis, é uma excelente decisão quando se busca celeridade. Ainda assim, a versão on-line precisa ser testada antes do início do estudo (usualmente são realizadas pequenas avaliações piloto), tanto para avaliar a compreensibilidade e facilidade de resposta quanto para avaliar os aspectos de programação e os bancos de dados gerados. Mesmo com esses cuidados, Zhang et al. 16 mostraram que as mesmas escalas de avaliação psicológica, cujas respostas foram obtidas em entrevistas face a face (utilizando-se CASI - Computer Assisted Self-Interview), telefônicas e on-line, apresentam diferentes vieses de aceitabilidade social (de acordo com a sensibilidade e aceitabilidade do tópico em estudo).

Assim, websurveys, como qualquer outro método de pesquisa, apresentam limitações relacionadas ao método, e seus resultados precisam ser interpretados levando-as em consideração. Ainda, da mesma forma que em outros métodos, em websurveys também é fundamental que sejam definidos claramente os objetivos gerais e específicos, as hipóteses a serem testadas e as análises estatísticas a serem realizadas a priori. Finalmente, é necessário que os aspectos éticos previstos para as pesquisas envolvendo seres humanos sejam rigorosamente respeitados, e que os novos desafios éticos sejam amplamente discutidos e avaliados 17 . Dois exemplos são os cuidados referentes ao anonimato e segurança dos dados. É importante que os pesquisadores, ao usar softwares comerciais, avaliem se o IP (ou outros dados que possam identificar os participantes como o CEP (Código de Endereçamento Postal), endereço postal e e-mail) é coletado e/ou armazenado junto com informações fornecidas pelos usuários e de que forma estas informações são comunicadas para os participantes e protegidas. Além disso, os servidores precisam ser confiáveis e seguros para que os dados não sejam obtidos ou modificados por terceiros. Existem também discussões sobre a forma e o tamanho do Termo de Consentimento Livre e Esclarecido (TCLE), visto que um dos potenciais problemas é que o TCLE seja entendido apenas como disclaimer, da mesma forma que vemos em muitos websites e Apps (seja pelas letras miúdas, posição na página ou extensão, sendo que, em relação à última, textos curtos podem não conter todas as informações necessárias e textos demasiadamente longos podem potencialmente fazer com que o usuário não o leia atentamente).

Nenhum desses tópicos constitui uma novidade na área. A literatura científica apresenta diferentes sugestões/soluções e os desafios para lidar com cada um dos aspectos citados e tantos outros que não foram, mas são igualmente relevantes. Todavia, garantir a integridade da Ciência em momentos tão complexos como o que estamos vivendo e ao mesmo tempo gerar informações que possam ser úteis para a saúde de todos de forma ágil, não é uma tarefa simples. Dificilmente websurveys bem- sucedidas podem ser realizadas por um único pesquisador e, muitas vezes, mais de um grupo de pesquisa precisam ser envolvidos no processo. Nossa capacidade de conexão, trabalho em equipe/redes e colaboração é fundamental nesse sentido e continuará sendo se quisermos gerar soluções criativas para o futuro próximo. Especificamente na área de comportamento e saúde mental, parte dessas soluções somente será possível ao conciliarmos nossa capacidade técnica com a tecnologia e boas estratégias de comunicação 18. Construir e reforçar essas pontes em prol do bem comum pode ser uma herança positiva desta crise sem precedentes. 


\section{Informação adicional}

ORCID: Raquel Brandini De Boni (0000-00022455-5997).

\section{Referências}

1. Marquez GG. O amor nos tempos do cólera. Rio de Janeiro: Editora Record; 1986.

2. Hewson C, Vogel C, Laurent D. Internet research methods. London: Sage Publishing; 2016.

3. Kaye BK, Johnson TJ. Research methodology: taming the cyber frontier. Techniques for improving online surveys. Soc Sci Comput Rev 1999; 17:323-37.

4. Solomon DJ. Conducting web-based surveys. Practical Assessment, Research, and Evaluation 2001; 7:19.

5. Sills $\mathrm{S}$, Song C. Innovations in survey research: an application of web-based surveys. Soc Sci Comput Rev 2002; 20:22-30.

6. Joncew CC, Cendon BV, Ameno N. Websurveys como método de pesquisa. Informação \& Informação 2014; 19:192-218.

7. Gallè F, Sabella EA, Da Molin G, Liguori G, Montagna MT, Orsi GB, et al. A cross-sectional study investigating lifestyle and weight perception of undergraduate students in southern Italy. BMC Public Health 2019; 19:1316.

8. Domche GN, Valois P, Canuel M, Talbot D, Tessier M, Aenishaenslin C, et al. Telephone versus web panel National Survey for monitoring adoption of preventive behaviors to climate change in populations: a case study of Lyme disease in Québec, Canada. BMC Med Res Methodol 2020; 20:78.

9. Chang L, Krosnick JA. National surveys via RDD telephone interviewing versus the internet: Comparing sample representativeness and response quality. Public Opin Q 2009; 73:64178.

10. Marcon G, Monteiro GMC, Ballester P, Cassidy RM, Zimerman A, Brunoni AR, et al. Who attempts suicide among medical students? Acta Psychiatr Scand 2020; 141:254-64.
11. Torres TS, Bastos LS, Kamel L, Bezerra DRB, Fernandes NM, Moreira RI, et al. Do men who have sex with men who report alcohol and illicit drug use before/during sex (chemsex) present moderate/high risk for substance use disorders? Drug Alcohol Depend 2020; 209:107908.

12. Núcleo de Informação e Coordenação do Ponto BR. TIC Domicílios 2019. https://cetic.br/ pesquisa/domicilios/indicadores/ (acessado em 28/May/2020)

13. Eysenbach G. Improving the quality of web surveys: the Checklist for Reporting Results of Internet E-Surveys (CHERRIES). J Med Internet Res 2004; 6:e34.

14. Fleming CM, Bowden M. Web-based surveys as an alternative to traditional mail methods. J Environ Manage 2009; 90:284-92.

15. Lee SA, Mathis AA, Jobe MC, Pappalardo EA. Clinically significant fear and anxiety of COVID-19: a psychometric examination of the Coronavirus Anxiety Scale. Psychiatry Res 2020; 290:113112.

16. Zhang XC, Kuchinke L, Woud ML, Velten J, Margraf J. Survey method matters: online/offline questionnaires and face-to-face or telephone interviews differ. Comput Human Behav 2017; 71:172-80.

17. Salganik MJ. Ethics. In: Salganik MJ, editor. Bit by bit: social research in the digital age. Princeton: Princeton University Press; 2018. p. 281-325.

18. Balanzá-Martínez V, Atienza-Carbonell B, Kapczinski F, De Boni RB. Lifestyle behaviours during the COVID-19 - time to connect. Acta Psychiatr Scand 2020; 141:399-400.

Recebido em 06/Jun/2020

Aprovado em 11/Jun/2020 